\title{
LA ÉTICA DEL CUIDADO PARA EL BIEN DE LA CASA COMÚN
}

\section{THE ETHICS OF CARE FOR THE GOOD OF THE COMMON HOUSE}

\author{
Juan Carlos Díaz*
}

Recepción: 22/Octubre/2016

Aprobación: 15/Marzo/2017

\section{RESUMEN}

El texto analiza a la luz de la encíclica Laudato Sii la lógica 'sagrada' del mercado y cómo ella alienta un ethos de la acumulación que el creyente cristiano está exigido a contravenir a partir del reconocimiento de la crisis de lo humano y del ethos del cuidado.

\section{PALABRAS CLAVE}

Crisis, Mercado, Religión, Ética, Cuidado

\begin{abstract}
The text analyzes, in light of the encyclical Laudato Sii, the sacred logic of the market and how it encourages an ethos of accumulation, which the Christian believer is required to disregard in the face of the recognition of the crisis of the human person and the ethos of care.
\end{abstract}

\section{KEY WORDS}

Crisis, Market, Religion, Ethics, Care 
En el momento actual, vivimos una profunda crisis del ser humano en su relación consigo mismo, pero a la vez y al mismo tiempo, una crisis del ser humano con su entorno humano y natural, acaso efecto de una crisis de su relación con la trascendencia. Es importante desde el inicio, precisar, de la mano de Adela Cortina (1996), qué se entiende por «crisis». La crisis es un fenómeno habitual que ocurre o acontece en cualquier persona o sociedad. La crisis pone en cuestión, en determinados momentos del desarrollo, bien de la biografía personal, bien del devenir histórico de una sociedad, aquellos valores que se tienen por vigentes y empieza a preguntarse cuáles merecen la pena conservar y cuáles dejar de lado. Todas las sociedades - y las personas - atraviesan este proceso, porque es una consecuencia inevitable del cambio social (Cortina, 1996, p 30). La crisis permite revisar los criterios de verdad y comprensión que dábamos por certeros y nos sugiere, en muchas ocasiones, reformularlos. ${ }^{1}$ Cuando una sociedad atraviesa una crisis, (puede decirse también que la crisis atraviesa a la sociedad) ocurre que, de acuerdo a cómo se la gestione, la sociedad salga mejor o peor. En este último caso, una sociedad empeora si no está atenta y no resiste - y acepta- los discursos que acompañan la crisis y de modo pasivo y fatal considera que nada se puede hacer.

En lo que sigue reflexiono sobre esta crisis, a la luz de Carta Encíclica Laudato Si' del Papa Francisco. Usaré la abreviación
LS seguido del numeral correspondiente cuando me refiera a este documento.

El numeral 56 de LS sostiene:

"(...) los poderes económicos justifican el actual sistema mundial, donde priman la especulación y la búsqueda de renta financiera que ignoran todo contexto $y$ los efectos sobre la dignidad humana y el medio ambiente. Esto manifiesta que la degradación ambiental y la degradación humana y ética están intimamente unidas. Muchos dirán que no tienen conciencia de realizar acciones inmorales, porque la distracción constante nos quita la valentía de advertir la realidad de un mundo limitado y finito. Por eso, hoy «cualquier cosa que sea frágil, como el medio ambiente, queda indefensa ante los intereses del mercado divinizados."

Este texto me permite trazar el siguiente itinerario. En primer lugar, esbozaré los rasgos de la llamada sacralización del mercado. En segundo lugar, veremos cómo la fe cristiana realiza un significativo aporte para atender el descuido de la dignidad humana y de la casa que habitamos. El documento se cierra con algunas reflexiones.

El mercado ocupa hoy la centralidad del ámbito de lo sagrado, desplazando a otros absolutos como la Religión, e incluso al Estado. El neoliberalismo ha dejado de ser una teoría o propuesta económica para instalarse como una lógica cultural global. ${ }^{2}$

1 Ciertamente no podemos sostener que ahora tenemos la verdad, sino que, por el contrario, y de manera razonable, podemos decir que tenemos una mejor visión, hasta ahora, del fenómeno o la crisis que nos aqueja.

2 Cualquiera que sea el problema a enfrentar - el cambio climático, la pobreza, la reforma educativa - el mercado se promueve a sí mismo como una respuesta eficaz. Contra la pobreza, el mercado tiene programas eficientes; para frenar el calentamiento global, hay salida...si se reciben incentivos adecuados; el acuerdo comercial bilateral, más rápido, impulsa el crecimiento económico tanto en el país como en el extranjero; la privatización, lo sabemos, va a resolver las ineficiencias gubernamentales. Estos argumentos, sin embargo, se basan en una mala comprensión de la economía y la historia. No estamos argumentando que los mercados sean siempre perniciosos. pueden ser muy útiles. Sin embargo, la observación de que el mercado es beneficioso siempre y en todas partes y que su regulación es mala se ha extendido de manera perniciosa. 
El Mercado, así con mayúsculas, se presenta como nuevo absoluto desde el cual se comprende el ser humano. De este modo remite en él una seria de consideraciones tales como: (a) ser ley suprema, extra societaria, a la que tanto individuos y sociedades han de someterse, en todos los niveles de la vida social; (b) poseer un carácter intrínseco regulador que compense sus propios desequilibrios, esto es el mercado se regula solo, sin necesidad de que intervengan otras fuerzas como el Estado o la sociedad civil, u otras esferas de comprensión como la justicia; (c) dotar de valor social a cuanto entra en su ámbito, desvalorizando todo aquello que no se inscribe en sus dominios, así se entiende que el quehacer humano que se realiza fuera del mercado laboral, por ejemplo, no es tal o que aquello que no es útil o rentable es baldío-; (d) finamente, incluso, erigirse como una ética, una manera de actuar, regida solo por el objetivo de conseguir la maximización de beneficios en el menor tiempo, sea en el plano de lo estrictamente material como en el de las relaciones sociales, sentimentales e incluso espirituales. Desde el Absoluto Mercado, palabras como competitividad, productividad, e incluso emprendimiento se convierten en reglas que deben orientar comportamientos, actitudes y hasta emociones, para conseguir el éxito personal, esto es bienestar, prosperidad y poder - económico y social - que cada individuo llegue a ser capaz de conquistar (Beaudin 1997: 110-112; Moreno 2001 y De Sousa Santos 2014: 10-12).

LS presenta desde el inicio una evaluación de esta situación, desde una perspectiva más amplia, que nos sugiere hacer dos consideraciones. El primer remite al hecho de que los principales problemas de nuestro tiempo asociados a la lógica del mercado - la energía, el cambio climático, el desempleo, la dinámica homogenizante de la globalización - - no pueden entenderse de manera aislada. A esta situación crítica se tiene que responder de un modo completo, que comprenda las interrelaciones, los presupuestos e impactos de los problemas apuntados. El Papa utiliza el término ecología integral para referirse al enfoque sistémico, y destaca la interdependencia de las cuestiones ecológicas, sociales y culturales, a través del respeto de formas de saber y valorar la vida y el entorno, como suelen hacer las culturas originarias.

Una segunda consideración tiene que ver con la constatación de que la lógica del mercado alienta dos ilusiones. La primera ilusión sostiene que es posible explotar recursos, de modo ilimitado y permanente. El modelo económico vigente está convencido de la creencia según la cual tal crecimiento es perpetuo y se lleva a cabo sin descanso alentando el consumo excesivo y una economía de descarte que utiliza, además, energía y recursos naturales, que desecha luego de exprimirla como residuo contaminado y/o próximo a agotarse. El Papa Francisco reconoce claramente el defecto fatal de esta idea del crecimiento perpetuo y utiliza palabras fuertes para condenarlo:

\section{"Ahora lo que interesa es extraer todo lo posible de las cosas por la imposición de la mano humana (...) De aquí se pasa fácilmente a la idea de un crecimiento infinito o ilimitado, que ha entusiasmado tanto a economistas, financistas $y$ tecnólogos. Supone la mentira de la disponibilidad infinita de los bienes del planeta, que lleva a «estrujarlo»" [LS 106]}

Tal fuerza extractiva que estruja al planeta requiere, además, del automatismo de procesos eficientes y de la homogenización del trabajo que simplifica procedimientos y reduce costos. Todo lo cual se consigue haciendo del ser humano 
una pieza de la maquinaria productiva. La revolución industrial que descansa sobre la configuración de un nuevo tipo de sociedad ha gestado un modo diferente de entender la persona humana ${ }^{3}$. La libertad de técnicas productivas, de contratación de trabajo, lejos de las amarras, pero también de las protecciones de los antiguos gremios, ha gestado una nueva sociedad, de mercado, que considera al ser humano como un ser de carencias y necesidades que busca la convivencia por sus propios intereses, y que, por ello, vende su fuerza laboral para alimentar una maquinaria de la cual él mismo es un engranaje.

La segunda ilusión concierne a la idea según la cual el crecimiento económico por si solo es sinónimo de bienestar y desarrollo. Es claro que durante el último medio siglo, el crecimiento económico ha sacado a cientos de millones de personas de la pobreza y ha mejorado las vidas de muchas más. Así, los defensores del modelo pueden decir que, gracias al mercado, millones de chinos, por ejemplo, han pasado de la pobreza a mejores condiciones de vida. El paso de un régimen maoísta a uno como el actual es, claro está, comparativamente beneficioso. ¿Pero realmente han mejorado las condiciones laborales? En este caso la transformación económica no ha ido acompañada de una liberalización política ¿Hacia dónde va el gigante chino en el terreno de lo político?

En su momento, Juan Pablo II hacía notar en la encíclica Centessimus annus (CA) las consecuencias de una forma de globalización dominada exclusivamente por el mercado y dejó claro que el capitalismo neoliberal no existiría sin sus propias formas de injusticia (42). Mucho antes, Pablo VI, en la Populorum Progressio (PP) tomaba distancia de las soluciones neoliberales a la crisis. Su condena a un sistema que considera la ganancia como "el motivo clave para el progreso económico, la competencia como la ley suprema de la economía, y la propiedad privada de los medios de producción como un derecho absoluto que no tiene límites y no acarrea la obligación social correspondiente" (PP 26) se mantiene vigente.

Aunque atractivo, el Sacro Mercado conduce a divergencias de crecimiento entre los países desarrollados y los que están en desarrollo; a una mayor desigualdad en la distribución del bienestar; a la precariedad laboral; a la degradación ecológica, etc. ${ }^{4}$ Todas ellas son razones suficientes para llevar a cabo no solo una crítica sino una seria reforma de la hegemonía de un modelo económico que parece no tener otra finalidad que expandirse de modo indefinido y que, al hacerlo, provoca exclusión.

Un modelo más amplio e inclusivo de desarrollo requiere nuevas medidas con las que los ciudadanos y los formuladores de políticas puedan evaluar el desempeño nacional. El Producto Bruto Interno (PBI) es el indicador predilecto para medir si un país avanza económicamente, pues representa el valor de la producción de todos los bienes y servicios, da cuenta del dinamismo económico y posibilidades de consumo de las personas y de ventas

3 La sociedad industrial se constituye de tres elementos: (a) la división del trabajo, que se expresa en la diversificación productiva, (b) la propiedad privada, medio que garantiza el intercambio de productos y (c) el interés propio. El libre mercado afirma que todos estaremos mejor si cada uno de nosotros actúa de modo egoísta.

4 Como ha señalado Yuval Harari, el mito capitalista según el cual el crecimiento económico es la respuesta a todos los problemas ha impregnado tanto nuestra cultura que ni siquiera somos conscientes de ello. Esa es la forma en que la crisis nos afecta. Cfr. Harari 2014: 230. 
para las organizaciones. Sin embargo, la fijación obsesiva en el crecimiento del PBI conlleva a una clara confusión entre fines y medios. El aumento de la riqueza es un medio para alcanzar un fin: el bienestar humano. El PBI, al excluir de su medición el grado de contaminación, delincuencia, corrupción, desigualdad, desempleo, presenta un panorama incompleto de las condiciones de vida en un territorio.

Por eso, de modo alternativo, el concepto de desarrollo humano tiene que entenderse como una expansión de la riqueza de la vida humana, en contraste con el aumento de la riqueza económica, factor este último que contribuye al bienestar pero que es incompleto en sí mismo. Así, el reto es ir más allá de la simple medición del PBI per cápita y hacer de la medición social - y ambiental -parte integral de la medición del desempeño y de los logros de un país.

"Para que surjan nuevos modelos de progreso, necesitamos «cambiar el modelo de desarrollo global», lo cual implica reflexionar responsablemente «sobre el sentido de la economía y su finalidad, para corregir sus disfunciones $y$ distorsiones». No basta conciliar, en un término medio, el cuidado de la naturaleza con la renta financiera, o la preservación del ambiente con el progreso. En este tema los términos medios son sólo una pequeña demora en el derrumbe (...) Un desarrollo tecnológico y económico que no deja un mundo mejor y una calidad de vida integralmente superior no puede considerarse progreso" (S 194)

La expansión de los medios de comunicación, la Internet, la multifacética globalización, contribuyen, cómo no, al progreso humano. Sin embargo, cuando el mercado va demasiado lejos y se descontrola y sacraliza el efecto más directo es el incremento de la desigualdad, concentrando el poder y la riqueza en un grupo reducido de personas, empresas y países que deja en los márgenes y en la exclusión a muchas demás.

La sacralización del mercado, en su núcleo se ha instalado como un ethos, como una práctica que no está allá afuera, en el mercado, sino que opera también en otras esferas de la vida social: hemos internalizado un modo de ver las cosas que privilegia la competencia, la búsqueda de beneficio al menor costo posible, o al mayor costo ajeno, a creer que tener más es mejor. En el corazón de esta cultura se encuentra lo que un teórico del desarrollo como Gilbert Rist (1992) ha llamado eficiencia abstracta, una creencia según la cual, más es sinónimo de mejor: mientras más tengamos de un servicio, de un bien o de un recurso, mejor nos encontraremos en términos de nuestras condiciones de vida. Se comprende entonces por qué el discurso económico dominante entiende "desarrollo" como 'crecimiento'.

Esta lógica no rige solo con las cosas, sino también con las personas consideradas o como medio o como obstáculos para los fines egoístas del individuo. El mercado se fundamenta en esta imbricación de egoísmo y ethos de la acumulación que tiene como principal efecto el descuido de la dignidad humana y el medio ambiente.

\section{"El ambiente humano y el ambiente natural se degradan juntos, y no podremos afrontar adecuadamente la degradación ambiental si no prestamos atención a causas que tienen que ver con la degradación humana y social" (LS 48)}

Esta lógica se nutre, además, de sus consecuencias imprevistas o externalidades, esos efectos colaterales e infames respecto de los cuales el crecimiento es indiferente. El ethos de la acumulación, digo, es ajeno e indiferente 
a las consecuencias que ocasiona. ¿Cómo responder a esta situación?

En el mundo contemporáneo encontramos varios tipos de respuesta. Una primera es la que plantea soluciones solidarias en la dinámica del mercado internacional. Christian Comeliau (1991) señala, por ejemplo, que el desarrollo es un objetivo legítimo puesto que es necesario para que mejoren las condiciones de vida de los países en vías de desarrollo, pero conviene controlar el sistema económico alentando, por ejemplo, que las empresas multinacionales transfieran tecnologías a dichos países o que los acuerdos internacionales sobre materias primas permitan una estabilización de los precios favorable a todas las partes (Comeliou, 1991, p.91).

Una segunda respuesta se encuentra en las prácticas de algunos movimientos sociales que no esperan nada de los poderosos y que han dejado de creer en la cooperación internacional. Estos movimientos se organizan a través de nuevos lazos sociales y formas alternativas para conquistar derechos, de los que no se gozaban o que fueron sustraídos, renunciando a la lógica de la acumulación, conforme a sus propias ideas, luchando para que se respeten sus decisiones, tomadas además por ellos mismos, al margen del sistema (Rist, Rahnema y Esteva 1992: 75; Rahnema 1990: 23-24; De Sousa Santos 2014: 27) No son grupos activistas, como se pudiera creer, sino grupos que realizan una ruptura epistémica con el modelo dominante. Estos movimientos han cambiado la manera de comprenderse a sí mismos y al entorno: no quieren ser víctimas ni marginales, sino personas que dejan a un lado la sumisión para confiar en sí mismas.

Una tercera respuesta es la del cristianismo, que nunca ha sido ajeno a esta situación. El Papa elabora una respuesta de raíces evangélicas que se encuentra en el numeral 220 y que es de una síntesis notable. El Papa sostiene que, Para el creyente, el mundo no se contempla desde fuera sino desde dentro.

En efecto, la invitación permanente del Evangelio nos pide transformar el mundo desde dentro. El creyente de hoy está invitado a no vivir una vida espiritual de espaldas al mundo y sus circunstancias. ${ }^{5}$ La transformación - el mensaje de salud para el entorno, para el ser humano mismo - se hace a modo de fermento o de sal, metáforas vigentes que expresan que la Iglesia no es ajena a la historia y que su tarea consiste en ofrecer al mismo tiempo un mensaje de esperanza en un mundo de incertidumbre como gestos firmes en favor del bien común. El Papa Francisco propone algunas pistas de esta acción transformadora. Las inequidades del mundo contemporáneo no pueden pasar inadvertidas para el cristiano (LS 90), por el contrario el creyente está exigido a ejercer el ethos del cuidado, un modelo alternativo que nos recuerda que el ser humano no existe para dominar sino para cuidar, de sí, de los demás, del entorno, otorgando

5 El Concilio Vaticano II señaló con pertinencia un peligro del que el cristiano puede ser presa fácil: «Se equivocan los cristianos que (...) consideran que pueden descuidar las tareas temporales, sin darse cuenta de que la propia fe es un motivo que les obliga al más perfecto cumplimiento de todas ellas según la vocación personal de cada uno. Pero no es menos grave el error de quienes, por el contrario, piensan que pueden entregarse del todo a la vida religiosa, pensando que ésta se reduce meramente a ciertos actos de culto y al cumplimiento de determinadas obligaciones morales. El divorcio entre la fe y la vida diaria de muchos debe ser considerado como uno de los más graves errores de nuestra época (...) No se creen, por consiguiente, oposiciones artificiales entre las ocupaciones profesionales y sociales, por una parte, y la vida religiosa por otra» (GS 43). 
sentido a su existencia y dejando de lado una concepción egoísta equivocada que "con otro ropaje, sigue dañando toda referencia común y todo intento por fortalecer los lazos sociales" que "da lugar a un estilo de vida desviado" (LS 101) alejado de lo humano y de la naturaleza.

"Si la crisis ecológica es una eclosión o una manifestación externa de la crisis ética, cultural y espiritual de la modernidad, no podemos pretender sanar nuestra relación con la naturaleza y el ambiente sin sanar todas las relaciones básicas del ser humano. Cuando el pensamiento cristiano reclama un valor peculiar para el ser humano por encima de las demás criaturas, da lugar a la valoración de cada persona humana, y así provoca el reconocimiento del otro. La apertura a un «tú» capaz de conocer, amar y dialogar sigue siendo la gran nobleza de la persona humana. Por eso, para una adecuada relación con el mundo creado no hace falta debilitar la dimensión social del ser humano y tampoco su dimensión trascendente, su apertura al «Tú» divino. Porque no se puede proponer una relación con el ambiente aislada de la relación con las demás personas y con Dios. Sería un individualismo romántico disfrazado de belleza ecológica $y$ un asfixiante encierro en la inmanencia" (LS 119)

Todo esfuerzo será estéril, insiste el Papa, si no procuramos también difundir este nuevo paradigma acerca del ser humano, la sociedad y la relación con la naturaleza. Necesitamos restaurar el supuesto cultural de que los seres humanos son valorados por lo que son, no lo que ellos producen. Tenemos que reconocer que el objetivo de la sociedad debe ser el florecimiento humano de una manera sostenible.
Apunto ahora dos reflexiones finales. En primer lugar, la llamada a la justicia nunca debe ser acallada en las actividades de la Iglesia. La invitación a la caridad nunca está lejos del mandato de justicia. El ser humano, alimentado por la autosuficiencia tecnológica, ha perdido la conciencia de sus límites como creatura.

"Hoy -dice el Papa- no podemos dejar de reconocer que un verdadero planteamiento ecológico se convierte siempre en un planteamiento social, que debe integrar la justicia en las discusiones sobre el ambiente, para escuchar tanto el clamor de la tierra como el clamor de los pobres" (LS 49).

La gran cuestión de la justicia, que aquí solo apuntamos y que merece sin duda un análisis exhaustivo, se juega en la disponibilidad de los creyentes cristianos para denunciar, proponer y mostrar la posibilidad de una alternativa al modelo que vivimos. La justicia que brota de la fe será novedad transformadora allí donde ella ya esté operando.

En segundo lugar, LS es un mensaje que se deriva de la fe cristiana aceptada por una comunidad de creyentes. Exigir un cumplimiento universal de esta doctrina supondría que la humanidad entera ha abrazado la fe cristiana, lo cual está lejos de ser realidad. Incluso suponiendo que en toda convicción religiosa, sea cual fuere su credo, se afirmase la dignidad y promoción del ser humano como consecuencia de la $\mathrm{fe}^{6}$, habría que aceptar que cada credo religioso bien puede tener formas distintas de fundamentación y de formulación de sus reclamos sociales. Proponer un mensaje social, desde el cristianismo tiene dos exigencias mínimas: La primera es cuestionar críticamente las creencias

6 LS sin duda transmite y suscribe los principios de la Enseñanza social cristiana: orientar la economía al servicio del bien común, trabajar a favor de la dignidad de las personas, exigir que el destino universal de los bienes está por encima de la propiedad. (Cf.LS 159, 228-231, 189-198). 
que sostienen el ethos de la acumulación y plantear otras creencias para desmontar el razonamiento de la economía actual y culturalmente dominante; la segunda exigencia tiene que ver con la coherencia de vivir este mensaje en el interior de la comunidad que lo propone. LS invita pues a repensar el propio cristianismo en el momento actual (LS 121). Ambas exigencias acaso son buenas pistas para salir mejor de la crisis.

\section{REFERENCIAS:}

BEAUDIN, Michel (1997) "El neoliberalismo como religión". En Selecciones de teología, Vol. 36, n 142 , pp. 109-118

CONCILIO VATICANO II (1965) Constitución Pastoral sobre la Iglesia en el Mundo Actual (Gaudium et Spes)

CORNELIAU, Christian (1991) Les relations Nord-Sud. París: La Découverte.

CORTINA, Adela (1996) "La regeneración moral de la sociedad y de la vida política". En Cuadernos de teología, nº , pp. 29-37.

DE SOUSA SANTOS, Boaventura (2014) Si Dios fuese un activista de los derechos humanos. Madrid: Trotta

FRANCISCO (2015) Laudato si': sobre el cuidado de la casa común. Lima: Paulinas HARARI, Yuval N. (2014) De animales a dioses. Una breve historia de la humanidad. Madrid: Debate
JUAN PABLO II (1991) Centessimus annus: sobre el desarrollo humano en la caridad y la verdad. Lima: Paulinas

MORENO, Isidoro (2001) Religión, Estado y mercado: los sacros de nuestro tiempo. Disponible en Libertades Laicas red iberoamericana por las libertades laicas. Recuperado de:

http://www.libertadeslaicas. org.mx/images/articulos/10/ 01/01/100101187.pdf

PABLO VI (1967) Populorum progressio: sobre el desarrollo de los pueblos. Lima: Paulinas

RAHNEMA, Majid (1990) "Swadhyaya: The unknown, the peaceful, the silent yet singing, revolution of India", pp. 19-34. En: Dossier de la Fundacion international para alternativas de desarrollo, 75-76, Enero-Abril. Recuperado de :

http://www.burmalibrary.org/docs 19/ ifda_dossier-75_76.pdf

RIST, G., RAHNEMA, M. y ESTEVA, G. (1992) Le Nord perdu, repères pour l'après développement. Lausanne, Editions d'En Bas

RIST, Gilbert (2002) El desarrollo: historia de una creencia occidental. Madrid: Catarata. 